\title{
On the Benefits of Channel Bonding in Dense, Decentralized Wi-Fi 4 Networks
}

\author{
Jose Manuel Gimenez-Guzman $\left(\mathbb{D},{ }^{1}\right.$ Ivan Marsa-Maestre $\left(\mathbb{D},{ }^{2}\right.$ David Orden $\left(\mathbb{D},{ }^{3}\right.$ \\ Susel Fernandez $\mathbb{D}^{2}{ }^{2}$ and Marino Tejedor-Romero $\mathbb{D}^{3}$ \\ ${ }^{1}$ Communications Department, Universitat Politècnica de València, Valencia, Spain \\ ${ }^{2}$ Computer Engineering Department, Universidad de Alcalá, Alcalá de Henares, Spain \\ ${ }^{3}$ Department of Physics and Mathematics, Universidad de Alcalá, Alcalá de Henares, Spain
}

Correspondence should be addressed to Jose Manuel Gimenez-Guzman; jmgimenez@upv.es

Received 7 July 2021; Revised 24 November 2021; Accepted 21 January 2022; Published 10 February 2022

Academic Editor: Ernestina Cianca

Copyright (c) 2022 Jose Manuel Gimenez-Guzman et al. This is an open access article distributed under the Creative Commons Attribution License, which permits unrestricted use, distribution, and reproduction in any medium, provided the original work is properly cited.

\begin{abstract}
Channel bonding is a technique first defined in the IEEE 802.11n standard to increase the throughput in wireless networks by means of using wider channels. In IEEE 802.11n (nowadays also known as Wi-Fi 4), it is possible to use $40 \mathrm{MHz}$ channels instead of the classical $20 \mathrm{MHz}$ channels. Although using channel bonding can increase the throughput, the classic 802.11 setting only allows for two orthogonal channels in the $2.4 \mathrm{GHz}$ frequency band, which is not enough for proper channel assignment in dense settings. For that reason, it is commonly accepted that channel bonding is not suitable for this frequency band. However, to the best of our knowledge, there is not any accurate study that deals with this issue thoroughly. In this work, we study in depth the effect of channel bonding in Wi-Fi 4 dense, decentralized networks operating in the $2.4 \mathrm{GHz}$ frequency band. We confirm the negative effect of using channel bonding in the $2.4 \mathrm{GHz}$ frequency band with 11 channels which are $20 \mathrm{MHz}$ wide (as in North America), but we also show that when there are 13 or more channels at hand (as in many other parts of the world, including Europe and Japan), the use of channel bonding yields consistent throughput improvements. For that reason, we claim that the common assumption of not considering channel bonding in the $2.4 \mathrm{GHz}$ band should be revised.
\end{abstract}

\section{Introduction and State of the Art}

The huge increase of wireless devices competing for the limited wireless bandwidth [1] has attracted the attention of researchers, since it is an increasingly complex problem. Especially in the case of the $2.4 \mathrm{GHz}$ band, where a greater number of devices and protocols coexist, and in dense, decentralized settings such as residential buildings, we find very inefficient bandwidth usage situations [2]. The community is addressing this challenge in a twofold manner. Some researchers focus on new standards and specifications for high-efficiency wireless local area networks (HEWs) [2]. Others, however, focus on improving the centralized or decentralized coordination of devices and networks using the existing standards.
In the latter case, channel assignment techniques aim to optimize the distribution of channels among the transmitting devices, thus decreasing interference and increasing throughput [3-8]. An additional possibility, which exists since the IEEE $802.11 \mathrm{n}$ standard (Wi-Fi 4), is the use of channel bonding, which consists of using channels that are wider than the standard $20 \mathrm{MHz}$ to achieve higher performance (higher bandwidth would allow for higher transmission rates, thus increasing throughput). A number of channel bonding techniques have been proposed in the literature for the different IEEE 802.11 standards [9-11]. We are especially interested in the standard IEEE $802.11 \mathrm{n}$ in the $2.4 \mathrm{GHz}$ band. However, the general consensus is that using channel bonding in the $2.4 \mathrm{GHz}$ band is not beneficial, since the interference due to the use of wider overlapping channels 
jeopardizes the theoretical advantage of having higher maximum bit rates [12]. Consequently, most studies assume other bands such as the $5 \mathrm{GHz}$ band [13-16], and the more recent ones generally assume dynamic channel bonding schemes for the 802.11ax standard [11,17].

Nevertheless, in our opinion, the possibilities of static channel bonding in the $802.11 \mathrm{n} 2.4 \mathrm{GHz}$ have not been properly analyzed in the literature. Since most of the papers mentioning the limitations of channel bonding in this band directly focus on other bands or technologies (i.e., the $2.4 \mathrm{GHz}$ band is not the focus of the paper), they either state these limitations as a matter of fact, not citing any study to back up the claim [18] or cite other papers which, in turn, state such limitations without the backup of an academic study $[12,15,16,19]$. There are some references to industrial white papers such as [20], but there the North American 11channel 802.11 spectrum is assumed, although there are many regions in the world (e.g., Europe or Japan) where more channels are available. Furthermore, some studies directly assume the use of orthogonal channels, and therefore they do not consider interferences between adjacent channels [21], or they use only one wireless station (STA) per access point (AP) [10], while it has been shown that both interference between adjacent channels and STA number and precise placement may have a significant effect on throughput [22]. Taking this into account, we believe that the aforementioned consensus about the goodness of channel bonding in the $2.4 \mathrm{GHz}$ band should be revised, as it is based on former studies [23-25] that concluded that channel bonding causes more harmful problems than it solves but these studies do not represent the density of current Wi-Fi networks.

In this paper, we study the effect of channel bonding in dense, decentralized Wi-Fi 4 scenarios, such as a residential building. The paper contributions can be summarized as follows:

(i) We describe a graph-based scenario model for $\mathrm{Wi}$ Fi 4 dense decentralized networks, using realistic indoor signal propagation and interference models, as well as the precise location and interference between all wireless devices (both access points (APs) and stations (STAs)), in order to compute the throughput. To the best of our knowledge, this is the first time that such an accurate model is used in the context of channel bonding (Section 2).

(ii) We provide a three-dimensional realistic setting for a decentralized Wi-Fi 4 deployment in a residential building. For this setting, we generate 60 scenarios for different STA densities and placements (Section 2.1).

(iii) We conduct an in-depth evaluation with the aforementioned model and setting, first for the classic 11-channel Wi-Fi 4 settings (as in, e.g., North America) and then for 13-channel Wi-Fi 4 (as in, e.g., Europe) (Sections 2.3 and 2.4).

Our results show that, on average, the use of channel bonding in an 11-channel Wi-Fi 4 setting yields a decrease in performance, although there may be some clusters of STAs reaping significant benefits at the expense of the others, which yields fairness concerns. This essentially matches the premises and conclusions in the consensus about channel bonding so far. However, for the 13-channel setting, our results show a consistent advantage of using channel bonding, contrary to the previous belief. The potential fairness issues remain, which opens interesting avenues for future work as we discuss in Section 4.

\section{Wi-Fi 4 Network Model}

2.1. Wi-Fi Networks. IEEE 802.11 networks, commercially known as $\mathrm{Wi}-\mathrm{Fi}$, are the most widespread technology to deploy wireless local area networks (WLANs). Although WiFi networks can operate in ad hoc and infrastructure modes, in this work we focus on the infrastructure mode, as it is the most widely used. In this operating mode, all the communications occur between access points (APs) and their associated stations (STAs), so if two STAs want to communicate to each other, this communication must go through an AP.

One of the main features of Wi-Fi networks is that this type of networks operates in unlicensed frequency bands. Among these frequency bands, we can highlight the spectrum around $2.4 \mathrm{GHz}$ and the spectrum around $5 \mathrm{GHz}$. Although the $5 \mathrm{GHz}$ band offers higher bandwidth and throughput, the $2.4 \mathrm{GHz}$ is still the most widely used frequency band due to its better coverage and its compatibility with more legacy equipment. To overcome the limitations in bandwidth, Wi-Fi standards have proposed the use of wider frequency channels, which is called channel bonding. More specifically, IEEE $802.11 \mathrm{n}$ (Wi-Fi 4) proposes the use of $40 \mathrm{MHz}$ channels instead of the classic $20 \mathrm{MHz}$ ones. Later standards open the possibilities of channel bonding to other bands and wider channel widths, up to $160 \mathrm{MHz}$. In this paper, we will focus on the Wi-Fi 4 standard in the $2.4 \mathrm{GHz}$ band.

2.2. Graph Modeling. To evaluate the effect of channel bonding in dense Wi-Fi networks, we make use of graph models that accurately represent the peculiarities of this type of networks. In fact, our models represent a set of independent Wi-Fi networks spatially distributed and modeled using geometric graphs. A graph can be defined as a set of vertices $V$ and a set of edges $E$ between them, $E \subseteq\{(u, v) \mid u, v \in V\}$. In our case, we consider geometric graphs, because the spacial positions of both APs and STAs (which will be the two kinds of vertices in our graphs) have a strong influence in the performance of the network [22]. In our graph model, we will also have two types of edges, one type representing the association between STAs and APs and the other type representing the interfering signals between wireless devices of different networks. To model interferences, we use an activity factor to account for the fact that STAs and APs do not transmit continuously, and we assume higher $\psi$ for APs. Although in this paper we consider 3D graphs, for the sake of an easier visualization, Figure 1 shows 


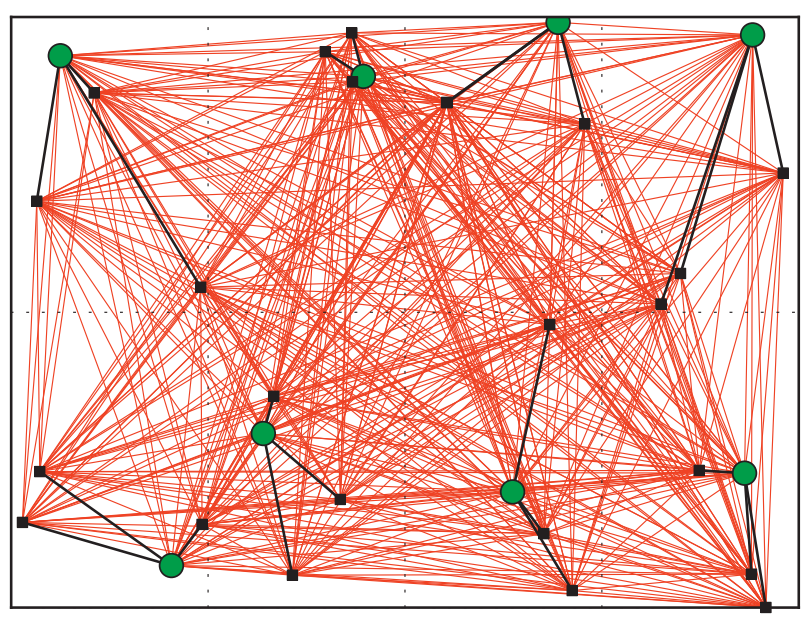

Figure 1: Example of Wi-Fi networks modeled with a graph.

a $2 \mathrm{D}$ example of the Wi-Fi layout for a single floor of a building, composed of 8 flats, 8 APs (one AP per flat), and 24 STAs (3 STAs per AP). We represent the APs as green circles and the STAs as black squares. Regarding edges, black segments represent the associations between APs and STAs, while red segments represent the interfering signals.

We have made use of a graph-based model for the following reasons. Although we have used discrete event simulators in the context of wireless networks in the past [26], we have noted that those papers that study dense Wi-Fi networks using simulation are difficult to replicate, especially because of the effect of the interferences between adjacent channels [27], which are not negligible at all in dense Wi-Fi settings. For that reason, we chose a graph model that can capture a high number of Wi-Fi network features (not with the precision of simulation models) but is faster and easier to replicate and to use by other researchers for comparison.

2.3. Propagation, Interferences, SINR, and Throughput Computation. Once we have the graph that represents the Wi-Fi layout, we must define how we can compute the achieved throughput for each STA. First of all, it is important to emphasize that the geometry of the problem defines the distances of the different $\mathrm{Wi}$-Fi elements, so with a proper propagation model, we will be able to compute the received signals from the different Wi-Fi elements, being either the desired signal or, mostly, interferences. As we focus on indoor Wi-Fi environments (dense Wi-Fi networks are usually indoor networks), we have used the propagation model defined by the ITU-R in the Recommendation P.1238-10 [28], as it assumes that STAs and APs are in the same building, which will be our testing scenario. Moreover, the ITU-R propagation model also considers losses across different building floors. In [28], propagation losses (in dB) are defined by

$$
L_{\text {total }}=20 \log _{10} f-28+N \log _{10} d+L_{f}(n),
$$

with $f$ being the frequency expressed in $\mathrm{MHz}, N$ the distance power loss coefficient, $d$ the distance in meters, and $L_{f}(n)$ the floor penetration factor when signal goes across $n$ floors. For the $2.4 \mathrm{GHz}$ frequency band, [28] defines $N=28$ in residential environments, although it is admitted that propagation through walls increases this value considerably. Therefore and according to [29], we have considered $N=28$ when $d<16$ meters and $N=38$ for $d \geq 16$ meters. Finally, according to [28], the losses across two floors when using concrete are $10 \mathrm{~dB}$, so we have considered $L_{f}(n)=10 n$.

After computing the propagation losses, we can compute the signal power (expressed in $\mathrm{dBm}$ ) received by an AP or STA $i$ from another Wi-Fi element $j$ as

$$
P_{r}^{j \longrightarrow i}=P_{t}^{j}+G_{j}+G_{i}-L_{\text {total }}
$$

where $P_{t}^{j}$ represents the transmission power of $j$ (in $\mathrm{dBm}$ ) and $G_{j}$ (or $G_{i}$ ) stands for the transmission (or reception) antenna gain (in $\mathrm{dB}$ ).

Next, we explain how we compute in the model the interferences received at a device $i$. In general, a device $i$ will receive interferences from all the transmitting devices in the whole network, excepting from the devices that belong to its same cluster, as their communications are coordinated and do not interfere. Note that a cluster defines the set made by an AP and all its associated STAs. The power of the interfering signal received at device $i$ from device $j$ $\left(I^{j \longrightarrow i}\right)$ will be the power of the received signal from $j$, i.e., $P_{r}^{j} \longrightarrow i$. However, the interference will only be relevant to the device $i$ to the extent that there is an overlap between the spectrum masks (in the frequency domains) as the communications between devices from the same cluster are coordinated and do not interfere. The model accounts for this overlap by means of parameter $\kappa$. If both channels are the same, we will consider a total overlap and $\kappa=1$. On the contrary, if both channels do not collide in the spectrum (orthogonal channels), we will have $\kappa=0$. Finally, if both channels partially collide, we will consider values of $\kappa$ ranging from 0 to 1 . In addition, we must also consider that, to account for the interference produced from device $j$ to device $i$, device $j$ is not making use of the spectrum continuously, from a temporal point of view. That behavior is considered by means of the activity factor $\psi$ introduced in Section 2, which can be either $\psi_{A P}$ or $\psi_{S T A}$ depending on whether the interfering source is an AP or a STA, respectively. In a sense, factor $\psi$ represents the CSMA/CA (Carrier Sense Multiple Access with Collision Avoidance) behavior of Wi-Fi networks. Some works [30] have modeled it as a Continuous Time Markov Chain (CTMC), concluding that when a STA or AP wants to transmit a packet with probability $P_{S T A}$ (or $P_{A P}$, respectively), it will succeed with probability $P_{s}$. For that reason, $\psi_{A P}$ can be computed as $\psi_{A P}=P_{A P} \cdot P_{s}$, and, equivalently, $\psi_{S T A}=P_{S T A} \cdot P_{s}$. As an AP is expected to transmit with a higher probability than STAs, $P_{A P}>P_{S T A}$, and therefore $\psi_{A P}>\psi_{S T A}$. In summary, we can compute the interference produced by a device $j$ to a device $i\left(I^{j \longrightarrow i}\right)$, in a linear scale, by considering the power of the received signal $\left(P_{r}^{j \longrightarrow i}\right)$, the frequency overlap of their transmission/reception channels $(\kappa)$, and the activity factor that accounts for the fraction of time during which the interference is being produced $(\psi)$ : 


$$
I^{j \longrightarrow i}=P_{r}^{j \longrightarrow i} \cdot \psi \cdot \kappa
$$

From the computation of the desired signal and all the interferences, for a specific STA, it is straightforward to compute the Signal-to-Interference-plus-Noise Ratio (SINR), as it is the quotient of the received power of the signal from its associated AP divided by the sum of the power of all the interfering signals plus the thermal noise. Note that the thermal noise depends on the channel bandwidth, so we consider its value to be $-101 \mathrm{dBm}$ for $20 \mathrm{MHz}$ channels and $-98 \mathrm{dBm}$ when using channel bonding (with $40 \mathrm{MHz}$ channels).

Finally, to compute the downlink throughput perceived by a STA, we must use the SINR together with the modulation and coding scheme (MCS) used. Depending on the SINR, Wi-Fi 4 [31] defines a specific MCS to be used, which in turn determines the throughput achieved by the STA. As the SINR is higher, it is possible to use modulations with a higher number of bits per symbol and coding schemes with less redundancy. These predefined MCSs, together with the throughput of each MCS for $20 \mathrm{MHz}$ and $40 \mathrm{MHz}$ channels, as defined in the standard [31], are shown in Table 1. Moreover, the table also shows the different SINR thresholds that determine the use of a specific MCS, according to [32].

2.4. Channel Assignment. One of the main configuration challenges in Wi-Fi networks is the choice of the channel to operate in, as defined in Section 1. There have been many works [3,33-35] focused on channel assignment for different Wi-Fi networks. However, channel assignment in uncoordinated Wi-Fi networks is usually based on a local decision based on using the channel where the perceived interference power is minimal [36], so this will be the channel assignment technique considered in this work. More specifically, in the channel selection algorithm, we have considered that each AP periodically scans the spectrum and chooses the channel where it detects the minimum power of interfering signals. This procedure operates asynchronously among the APs changing the order in which the different APs scan the environment. Note that this channel selection procedure represents the usual situation when a user sets up his/her AP leaving the channel selection to a decision of the AP, typically using the option called "Auto" instead of forcing the use of a specific channel. Moreover, as is commonly accepted and as has been suggested in previous works like [22], we restrict the possible channels to be used to the orthogonal channels, so they do not interfere with each other. However, the width of the $2.4 \mathrm{GHz}$ in North America does not allow to use two $40 \mathrm{MHz}$ channels that are totally orthogonal (i.e., nonoverlapping), so we have considered a case where there is some interference between the most separate channels in the spectrum. This will be described in detail in Section 3.3.

\section{Performance Evaluation}

In this section, we provide an in-depth evaluation of channel bonding in Wi-Fi 4 when operating in the $2.4 \mathrm{GHz}$ frequency band. After a description of the real-world model we have considered, we perform a validation of the model using the well-known ns-3 simulator [37]. Then, we study channel bonding in two different settings. First, we consider the spectrum that can be used in North America, consisting of 11 channels with $20 \mathrm{MHz}$ width each. For the sake of simplicity, we will name this setting $2.4 \mathrm{GHz} U S A$. Second, we consider the setting where there are 13 possible channels in the frequency band, as in many parts of the world including Europe. We will name this last setting $2.4 \mathrm{GHz}$ Europe. Finally, we conduct an analysis of fairness when using channel bonding.

3.1. Experimental Setting. The evaluation of channel bonding has been performed in a three-dimensional realistic setting that represents a five-floor residential building. This scenario is a typical example of a dense uncoordinated Wi-Fi 4 network. The dimensions of the building are $40 \times 30 \times 15$ meters (respectively, length, width, and height; thus, each floor has a height of 3 meters). Each floor has 8 different flats in a $4 \times 2$ arrangement. Regarding the distribution of Wi-Fi networks, we consider that each flat has a single AP and a number $\eta$ of STAs attached to that AP. Note that all the STAs from a flat are attached to the AP from the same flat, which can be the closest AP or not. Moreover, we have considered a wide range of density of STAs in this setting, ranging from $\eta=1$ STA per AP to $\eta=12$ STAs per AP. The position of each AP and associated STAs is limited to its flat, with its position in the $x$ - and $y$-axis being randomly distributed according to a uniform distribution. However, in the $z$-axis, each AP and each STA is randomly distributed with a normal distribution with a mean of 1.5 meters and a standard deviation of 0.5 meters, bounded to the limits of the floor. To sum up, all the scenarios under study consist of $8 \times 5=40$ flats and their corresponding 40 APs and a number of STAs ranging from 40 (when $\eta=1$ ) to $12 \times 40=480$ (when $\eta=12$ ). Finally, for each specific layout, we have considered 5 different settings to account for the randomness in the deployment of the different Wi-Fi elements, for a total of 60 scenarios. Figure 2 shows a graphical representation of two of the scenarios under study, where, for the sake of clarity, we only show the association between APs and STAs.

Finally, Table 2 defines the values used for the main parameters needed to compute the throughput, which in all cases are typical or reasonable values.

3.2. Model Validation. For validation purposes, in this section we include a comparative evaluation of the results obtained using our proposed model with respect to the equivalent results obtained using a discrete event simulator. More specifically, we have chosen the well-known ns-3 simulator [37]. The reference setting for this validation consists of a single AP and a single STA (attached to that AP) positioned at different distances. As our model computes the highest reachable throughput that a STA is able to obtain, in the simulator we have considered a greedy traffic source that emits UDP datagrams with a rate higher than the maximum throughput that the technology permits. To make the results 
TABLE 1: Relation between MCS, SINR, and throughput in Wi-Fi 4 with mandatory $800 \mathrm{~ns}$ guard interval (GI) [31].

\begin{tabular}{lccccc}
\hline MCS index & Modulation scheme & Coding rate & Throughput for $20 \mathrm{MHz}(\mathrm{Mbit} / \mathrm{s})$ & $\begin{array}{c}\text { Throughput for } 40 \mathrm{MHz}(\mathrm{Mbit} / \mathrm{s}) \\
\text { SINR range } \\
(\mathrm{dB})[32]\end{array}$ \\
\hline 0 & BPSK & $1 / 2$ & 6.5 & 13.5 & {$[6.8,7.9)$} \\
1 & QPSK & $1 / 2$ & 13.0 & 4.0 & {$[7.9,10.6)$} \\
2 & QPSK & $3 / 4$ & 19.5 & 54.0 & {$[10.6,13.0)$} \\
3 & 16-QAM & $1 / 2$ & 26.0 & 81.0 & {$[13.0,17.0)$} \\
4 & 16-QAM & $3 / 4$ & 39.0 & 108.0 & {$[17.0,21.8)$} \\
5 & 64-QAM & $2 / 3$ & 52.0 & 121.5 & {$[21.8,24.7)$} \\
6 & 64-QAM & $3 / 4$ & 65.5 & 135.0 & \\
7 & 64-QAM & $5 / 6$ & & 24.0 & $28.1)$ \\
\hline
\end{tabular}

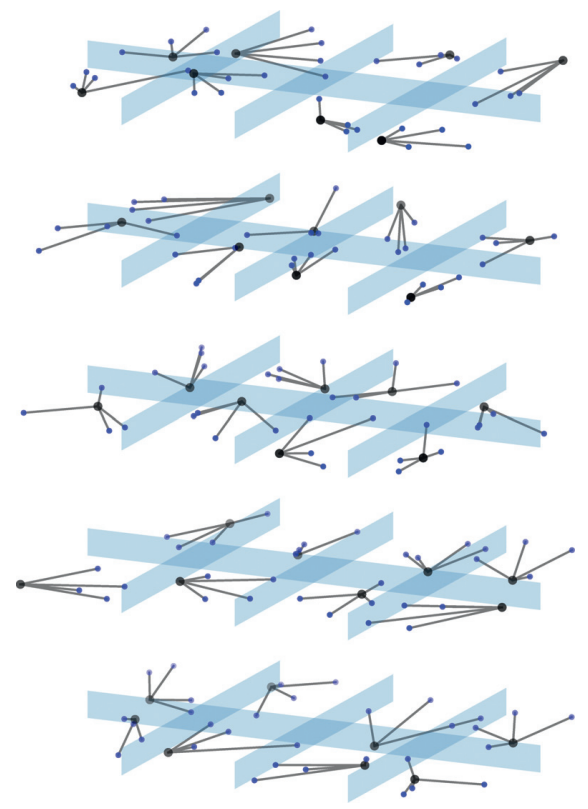

(a)

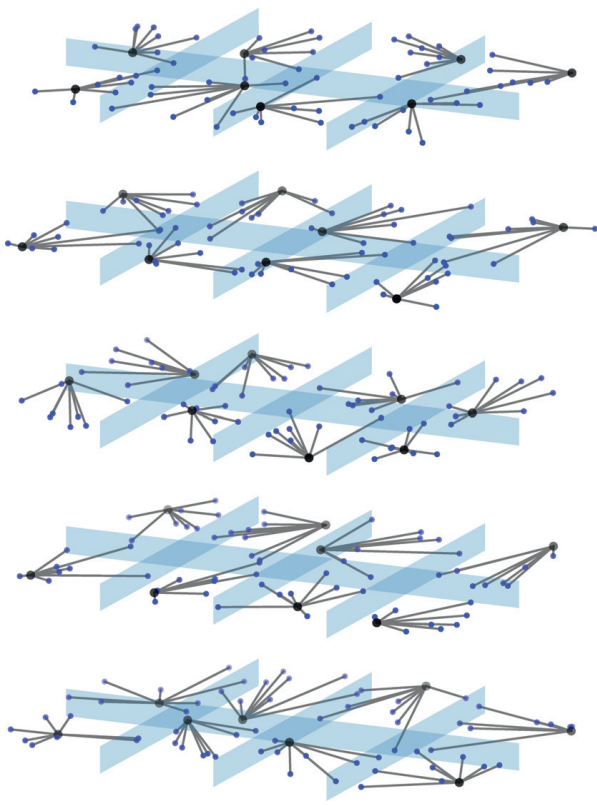

(b)

Figure 2: Examples of scenarios. (a) $\eta=4$. (b) $\eta=8$.

TABLE 2: Summary of parameters.

\begin{tabular}{lc}
\hline Parameter & Value \\
\hline$P_{t}$ & $30 \mathrm{~mW}$ \\
$G_{t}$ & $0 \mathrm{~dB}$ \\
$G_{r}$ & $0 \mathrm{~dB}$ \\
$\Psi_{A P}$ & 0.5 \\
$\Psi_{S T A}$ & 0.1 \\
\hline
\end{tabular}

comparable, we have used in ns-3 the same indoor propagation model, i.e., ITU-R P.1238-10, and we have configured the Wi-Fi manager in the simulator according to the settings used in our experiments.

The validation of our model has been conducted in a two-step procedure. First, at the physical level, we compare the received SINR, as is shown in Figure 3. We can see that both curves totally coincide, validating that our model and the ns-3 simulator obtain the same SINR. As a second step, we study the throughput obtained by the STA with our model and the simulator, as is shown in Figure 4. In that figure, we notice that the shapes of the curves coincide in both cases. However, there is a clear offset between both

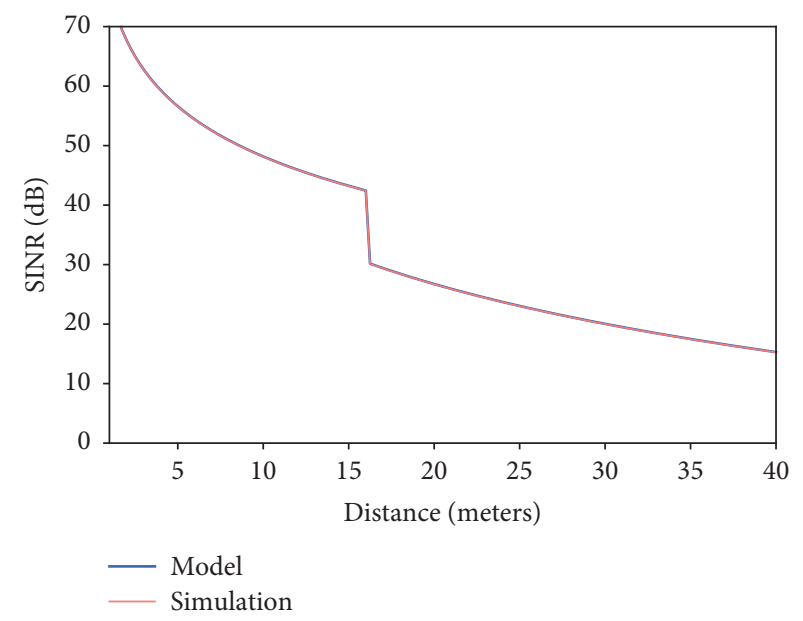

FIGURE 3: SINR obtained by our proposed model and by the ns-3 simulator.

curves. This behavior is due to the fact that our model measures the physical throughput, while ns-3 computes the throughput at the application layer (usually called goodput), 


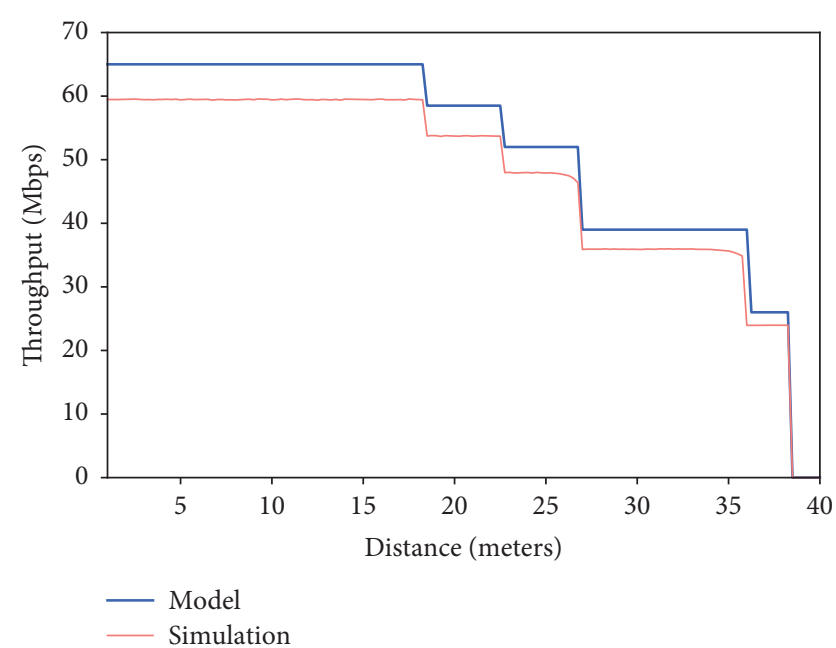

FIgURE 4: Throughput obtained by our proposed model and by the ns-3 simulator. Note. The model gives throughput at the physical layer, while the simulator measures goodput (application layer throughput).

with the difference between both values being the overhead introduced by the different layers of the protocol stack. That is the reason of measuring a higher throughput in our model, although both throughput measures coincide.

\subsection{Channel Bonding in the $2.4 \mathrm{GHz}$ USA Frequency Band.} In this section, we evaluate the effect that channel bonding has on the throughput perceived by users when they operate in the $2.4 \mathrm{GHz}$ frequency band with 11 nonorthogonal channels, which is the situation occurring in North America. In this setting, when using $20 \mathrm{MHz}$ channels, there are three different orthogonal channels, being channels 1,6 , and 11 . However, since the spectrum band goes from $2401 \mathrm{MHz}$ to $2473 \mathrm{MHz}$, we cannot use two different $40 \mathrm{MHz}$ orthogonal channels. Therefore, by placing one $40 \mathrm{MHz}$ channel in the lowest part of the spectrum and another $40 \mathrm{MHz}$ channel in the highest part, both channels will collide in the frequency band between 2433 and $2441 \mathrm{MHz}$. For that reason, we have considered that the interference index $(\kappa)$ when using two $40 \mathrm{MHz}$ channels in the $2.4 \mathrm{GHz}$ USA frequency band is $(2441-2433) / 40=0.2$. In other words, both channels, as they cannot be orthogonal, collide with a factor of $\kappa=0.2$, producing interferences to each other. Finally, for the sake of completeness, we have also considered the situation where we only use one $40 \mathrm{MHz}$ channel in the $2.4 \mathrm{GHz}$ USA frequency band, since this is the only possibility for totally orthogonal channels in this setting. Figure 5 shows the average downlink throughput and $95 \%$ confidence intervals that users can achieve when we consider either three orthogonal $20 \mathrm{MHz}$ channels, two (nonorthogonal) $40 \mathrm{MHz}$ channels, or one orthogonal $40 \mathrm{MHz}$ channel. For each value of $\eta$, the average throughput has been computed averaging the five different deployments that we have considered for each value of $\eta$ and running 10 executions for each of those five deployments. The rationale for performing 10 runs for each setting is that channel assignment technique used (as described in Section 3.4) is not deterministic (except when we make use of a unique $40 \mathrm{MHz}$

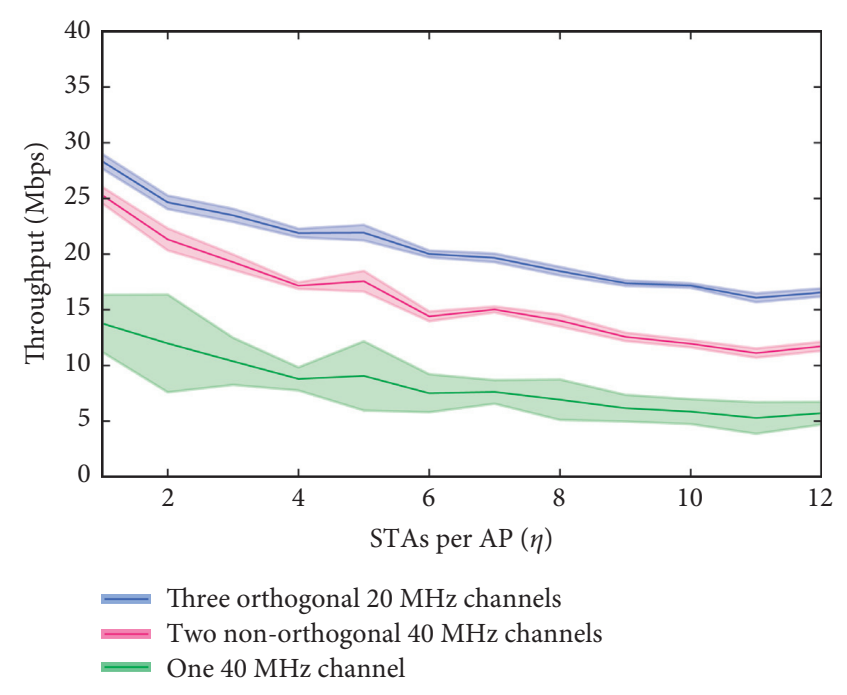

FIGURE 5: Comparison of average throughput in the $2.4 \mathrm{GHz}$ USA frequency band.

channel). As it could be expected, the achieved throughput decreases as the density of STAs $(\eta)$ increases. Moreover, results show that, on average, in the $2.4 \mathrm{GHz}$ USA frequency band, it is not recommended that channel bonding is used, as the gain that can be obtained by using channels with a higher bandwidth has a lower effect than that of having a fewer number of orthogonal channels, even when the density of users is low $(\eta=1)$. Additionally, we can also conclude that it would be better to use two $40 \mathrm{MHz}$ channels (although they are not completely orthogonal) than to use only one $40 \mathrm{MHz}$ channel.

Now, we perform a more in-depth analysis of the throughput that STAs can achieve individually. In Table 3 we show, for the different densities of STAs $(\eta)$ under consideration, the percentage of STAs that increase $(+)$, keep the same (=), or decrease (-) their downlink throughput when using channel bonding with two nonorthogonal $40 \mathrm{MHz}$ channels, compared to the situation where three orthogonal $20 \mathrm{MHz}$ channels are used. Moreover, the table also shows the average increase (or decrease) in throughput for the STAs that get better (or worse) performance when using channel bonding with two $40 \mathrm{MHz}$ channels. Inspecting Table 3, we observe that the percentage of STAs that improve their throughput is lower than the percentage of STAs that get a worse throughput, even with the lowest density of STAs, where only $26.5 \%$ of STAs fare better in terms of bandwidth and $65 \%$ fare worse. This confirms the common belief that, in dense scenarios, it is not recommended that channel bonding is used in the $2.4 \mathrm{GHz}$ USA frequency band. In addition, Table 3 shows that, even when the majority of STAs get a worse throughput, the average gain for the "improving" STAs is higher than the loss for the "losing" STAs, which can create fairness issues and misalignment of incentives in network management for these settings. Since the use of channel bonding is local to APs, the managers of some of the networks could unilaterally decide to transmit in $40 \mathrm{MHz}$ channels at the expense of the networks nearby. Even for those networks which, on average, "lose" throughput by using channel bonding, the fact that when the 
TABLE 3: Percentage of STAs that increase $(+) /$ keep $(=) /$ decrease $(-)$ their throughput (and average increase/decrease) using channel bonding with two nonorthogonal $40 \mathrm{MHz}$ channels in the $2.4 \mathrm{GHz}$ USA setting.

\begin{tabular}{lccccc}
\hline & \multicolumn{3}{c}{ \% STA } & \multicolumn{3}{c}{$\begin{array}{c}\text { Increase/decrease } \\
\text { (Mbps) }\end{array}$} \\
$\eta$ & & & & Increase & Decrease \\
\hline 1 & 26.50 & 8.50 & 65.00 & 20.29 & -13.27 \\
2 & 23.75 & 7.75 & 68.50 & 20.42 & -12.49 \\
3 & 21.83 & 9.50 & 68.67 & 19.00 & -12.36 \\
4 & 18.38 & 9.25 & 72.38 & 19.79 & -11.84 \\
5 & 17.90 & 11.10 & 71.00 & 20.02 & -11.56 \\
6 & 15.83 & 13.08 & 71.08 & 17.59 & -11.90 \\
7 & 16.29 & 14.36 & 69.36 & 17.70 & -11.08 \\
8 & 14.75 & 13.88 & 71.38 & 17.51 & -10.45 \\
9 & 13.56 & 15.83 & 70.61 & 17.31 & -10.89 \\
10 & 12.95 & 17.40 & 69.65 & 17.66 & -10.89 \\
11 & 12.64 & 18.45 & 68.91 & 15.95 & -11.05 \\
12 & 12.67 & 18.88 & 68.46 & 17.15 & -10.48 \\
\hline
\end{tabular}

load in the neighboring networks is low, the effective throughput is higher (because of using more bandwidth) may make managers choose to use channel bonding, thus hampering average performance for the network.

We now perform a similar analysis for the comparison of the gain of using one $40 \mathrm{MHz}$ channel with respect to using three $20 \mathrm{MHz}$ orthogonal channels (Table 4). As expected, the use of only one $40 \mathrm{MHz}$ channel is not recommendable, and the number of users that can improve their throughput is very low, ranging from $8 \%(\eta=1)$ to $2.73 \%(\eta=10)$. We see again that the average throughput increase for "winning" STAs is higher than the decrease for "losing" STAs, but this difference is not as remarkable as in the case of two nonorthogonal $40 \mathrm{MHz}$ channels.

Finally, Figures 6 and 7 show a ridge plot to evaluate the difference in the throughput that each STA can obtain when using two nonorthogonal $40 \mathrm{MHz}$ channels instead of three orthogonal $20 \mathrm{MHz}$ channels (Figure 6) or one $40 \mathrm{MHz}$ channel instead of three orthogonal $20 \mathrm{MHz}$ channels (Figure 7). Note that both figures represent the probability density functions expressed as a Kernel Density Estimation (KDE). In both figures, we observe that the number of STAs that decrease their throughput when using channel bonding (the area to the left of the vertical line at 0 ) is higher than the number of STAs that improve their throughput (the area to the right). Moreover, the tail on the right side of each KDE is longer than the tail on the left side, reflecting that there are STAs which greatly increase their throughput with channel bonding. Finally, inspecting the figures as the density of the $\mathrm{Wi}-\mathrm{Fi}$ scenarios increases (i.e., moving vertically from bottom to top), we conclude that channel bonding in the 2.4 $\mathrm{GHz}$ USA frequency band is even a worse choice when the density of the Wi-Fi network increases.

3.4. Channel Bonding in the $2.4 \mathrm{GHz}$ Europe Frequency Band. In this section, we perform a similar evaluation to the one provided in the previous section, but now focusing on the 2.4 GHz frequency band where there are at least thirteen
TABle 4: Percentage of STAs that increase (+)/keep (=)/decrease $(-)$ their throughput (and average increase/decrease) using channel bonding with one $40 \mathrm{MHz}$ channel in the $2.4 \mathrm{GHz}$ USA setting.

\begin{tabular}{lccccc}
\hline & \multicolumn{3}{c}{ \% STA } & \multicolumn{3}{c}{$\begin{array}{c}\text { Increase/decrease } \\
(\mathrm{Mbps})\end{array}$} \\
& & & - & Increase & Decrease \\
\hline 1 & 8.00 & 8.50 & 83.50 & 23.38 & -19.57 \\
2 & 7.25 & 7.75 & 85.00 & 24.07 & -17.13 \\
3 & 6.17 & 9.50 & 84.33 & 22.51 & -17.27 \\
4 & 5.13 & 9.25 & 85.63 & 21.63 & -17.11 \\
5 & 4.70 & 11.10 & 84.20 & 22.60 & -16.89 \\
6 & 4.33 & 13.00 & 82.67 & 17.40 & -16.23 \\
7 & 4.00 & 14.21 & 81.79 & 20.47 & -16.06 \\
8 & 3.31 & 13.81 & 82.88 & 19.12 & -15.15 \\
9 & 3.17 & 15.78 & 81.06 & 19.46 & -15.26 \\
10 & 2.70 & 17.30 & 80.00 & 20.46 & -15.07 \\
11 & 2.73 & 18.36 & 78.91 & 14.88 & -14.98 \\
12 & 2.83 & 18.88 & 78.29 & 16.74 & -14.81 \\
\hline
\end{tabular}

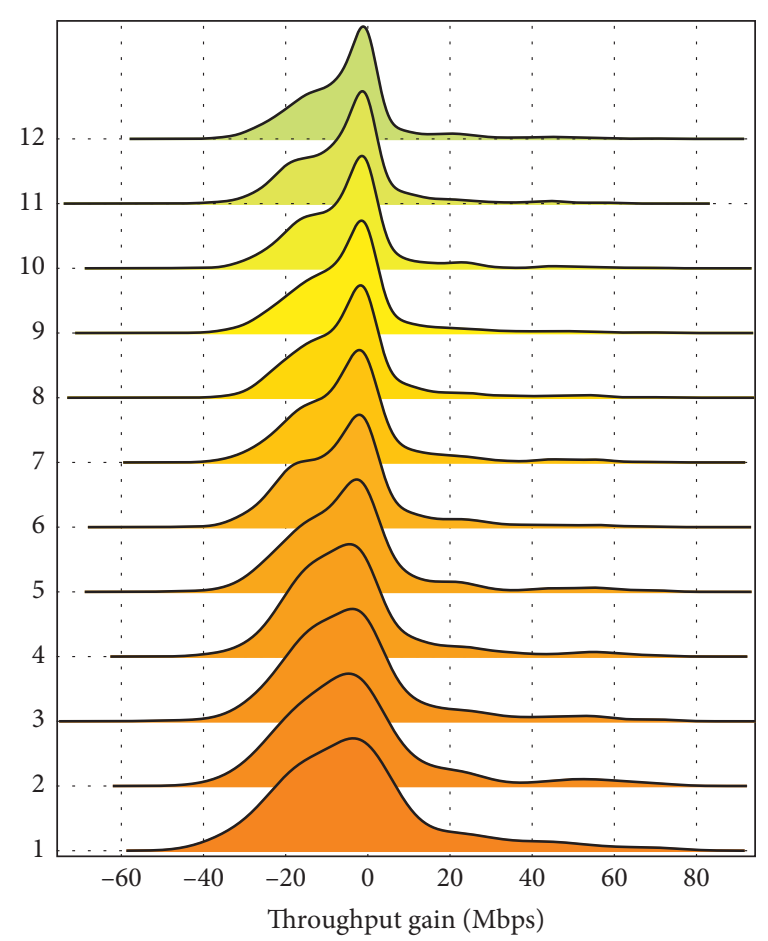

FIGURE 6: Density of the throughput gain of using channel bonding with two $40 \mathrm{MHz}$ channels in the $2.4 \mathrm{GHz}$ USA setting.

$20 \mathrm{MHz}$ channels available, as in most of the world and in particular in Europe. In this case, it is possible to use two $40 \mathrm{MHz}$ orthogonal channels, so we will compare two situations: using two orthogonal $40 \mathrm{MHz}$ channels and using three orthogonal $20 \mathrm{MHz}$ channels. Figure 8 shows the average downstream throughput achieved by STAs in both situations. As opposed to the behavior of the $2.4 \mathrm{GHz}$ USA frequency band, in this $2.4 \mathrm{GHz}$ Europe frequency band, the average throughput achieved increases when using channel bonding. Although the advantage of using channel bonding diminishes with the density of STAs $(\eta)$, it is always advantageous even in the more dense scenarios. 


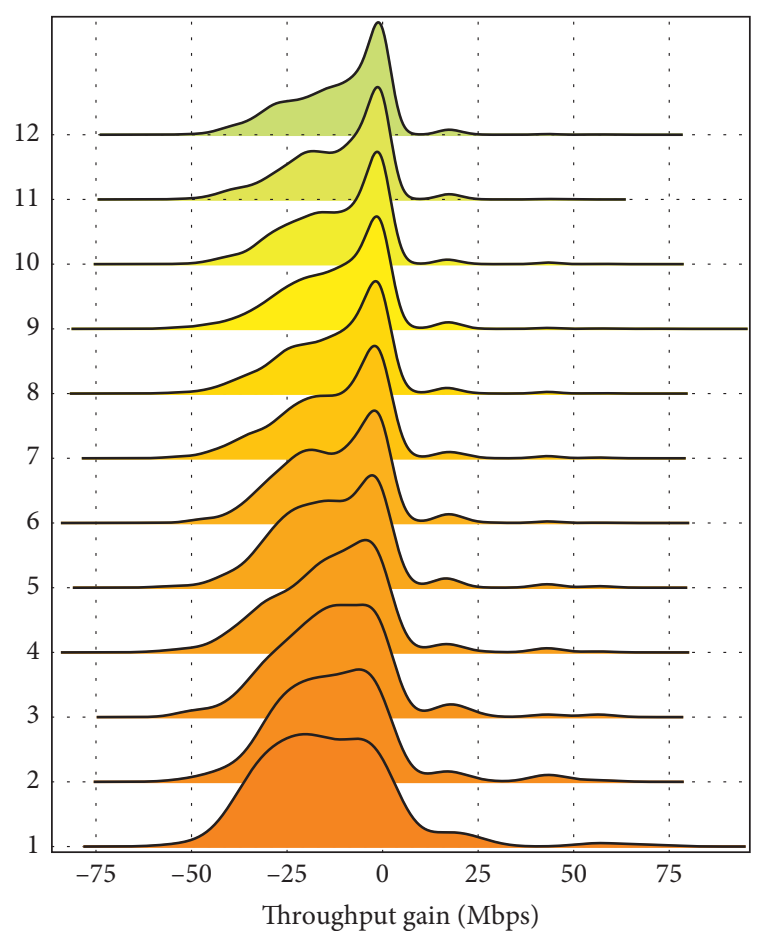

FIGURE 7: Density of the throughput gain of using channel bonding with one $40 \mathrm{MHz}$ channel in the $2.4 \mathrm{GHz}$ USA setting.

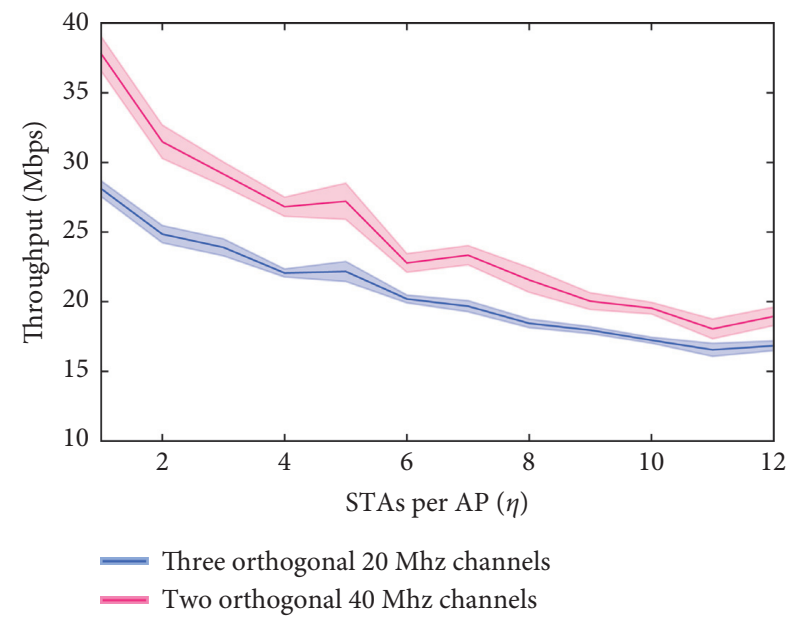

Figure 8: Comparison of average throughput in the $2.4 \mathrm{GHz}$ Europe frequency band.

Table 5 shows the number of STAs that are able to increase their throughput when using channel bonding. In general, for the lowest values of $\eta$, the percentage of STAs that increase their throughput is higher than the percentage of STAs that decrease it, but this is not true when the density of STAs increases. Furthermore, the average throughput gain of those STAs that improve their throughput is remarkably higher (around 19 to $23 \mathrm{Mbps}$ ) than the decrease of those STAs that get a worse throughput (around 6.5 to $9 \mathrm{Mbps}$ ).

Finally, in Figure 9, we show the KDE of the difference in the throughput perceived by STAs when using channel bonding, where we can remark the longer tails on the right of
TABle 5: Percentage of STAs that increase $(+) /$ keep $(=)$ /decrease $(-)$ their throughput (and average increase/decrease) with two orthogonal $40 \mathrm{MHz}$ channels in the $2.4 \mathrm{GHz}$ Europe setting.

\begin{tabular}{lccccc}
\hline & \multicolumn{3}{c}{ \% STA } & \multicolumn{3}{c}{$\begin{array}{c}\text { Increase/decrease } \\
(\mathrm{Mbps})\end{array}$} \\
$\eta$ & & & - & Increase & Decrease \\
\hline 1 & 55.00 & 8.50 & 36.50 & 23.52 & -9.03 \\
2 & 45.75 & 7.75 & 46.50 & 22.42 & -8.20 \\
3 & 44.33 & 9.33 & 46.33 & 20.48 & -7.33 \\
4 & 41.38 & 9.25 & 49.38 & 20.25 & -7.24 \\
5 & 41.30 & 11.10 & 47.60 & 19.77 & -6.72 \\
6 & 34.33 & 13.08 & 52.58 & 20.68 & -7.05 \\
7 & 35.36 & 14.21 & 50.43 & 19.93 & -6.59 \\
8 & 32.31 & 13.94 & 53.75 & 20.31 & -6.10 \\
9 & 32.11 & 15.72 & 52.17 & 18.99 & -6.56 \\
10 & 28.50 & 17.40 & 54.10 & 19.50 & -6.38 \\
11 & 31.14 & 18.23 & 50.64 & 18.39 & -6.66 \\
12 & 28.96 & 18.88 & 52.17 & 19.03 & -6.48 \\
\hline
\end{tabular}

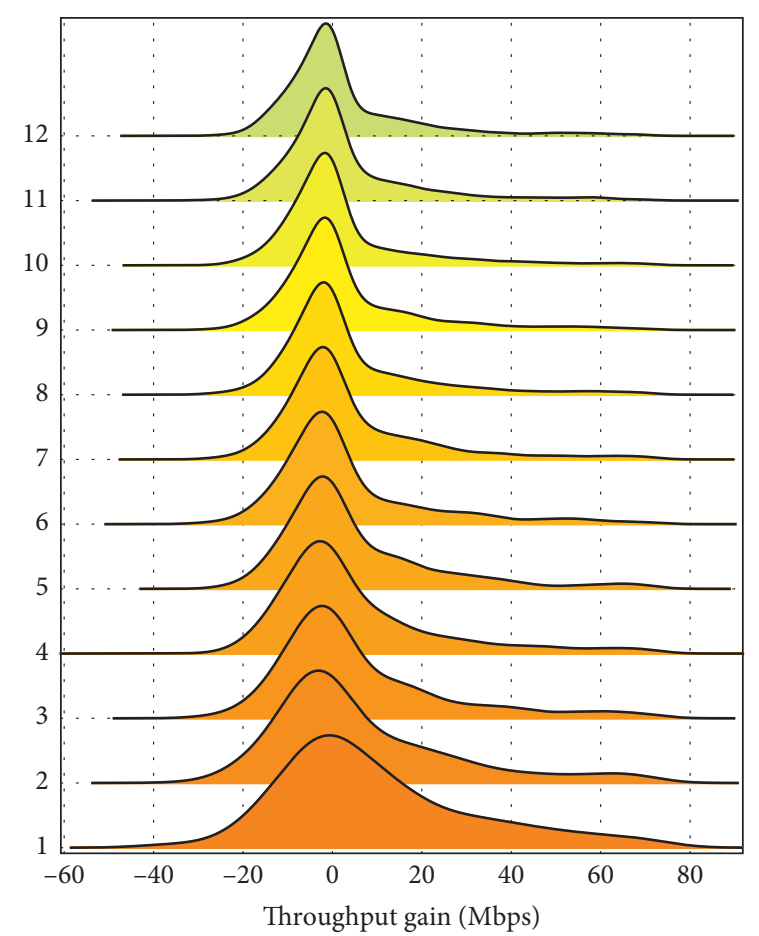

FIGURE 9: Density of the throughput gain of using channel bonding with two orthogonal $40 \mathrm{MHz}$ channels in the $2.4 \mathrm{GHz}$ Europe setting.

the different curves, which shows that the gain of using channel bonding for the "winning" STAs is higher than the loss in throughput for the "losing" ones. Taking this into account, we can conclude that, even when there are a nonnegligible number of STAs that decrease their throughput when using channel bonding, the high increase in an important fraction of STAs makes channel bonding in the $2.4 \mathrm{GHz}$ Europe frequency band worth using.

3.5. Study of Fairness. After the study of the throughput and the incentives that STAs can obtain by using channel 


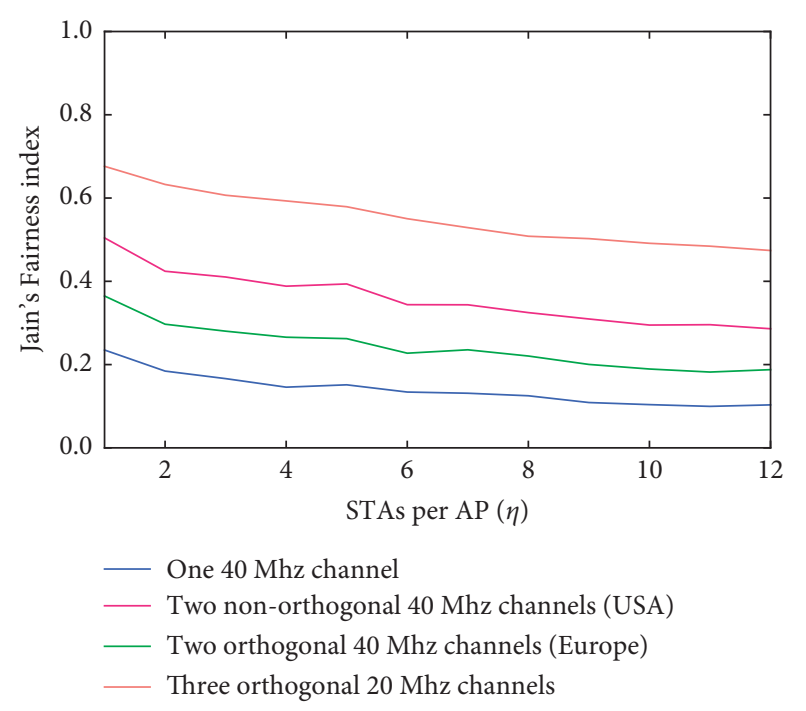

Figure 10: Study of fairness.

bonding in different regions, we complete the analysis of channel bonding by studying the fairness of the perceived throughput of the different STAs. The focus is to determine if channel bonding has an effect on fairness. The performance parameter used to measure fairness is the well-known Jain's fairness index [38], defined by

$$
f(x)=\frac{\left[\sum_{i=1}^{n} T h_{i}\right]^{2}}{\sum_{i=1}^{n} T h_{i}^{2}}, \quad T h_{i} \geq 0,
$$

with $T h_{i}$ being the downlink throughput perceived by STA $i$. Jain's fairness index is able to measure the "quality" of the service experienced by the different STAs. If all STAs obtain the same throughput, the fairness index is equal to 1 . As the disparity increases, the fairness index goes down to 0 , when the system clearly favors selected few users over the rest.

In Figure 10, we show the fairness for the different settings under study. Note that each value of a curve is the average value of the 5 different settings and 10 different executions. Results show that the best fairness is obtained when using three orthogonal $20 \mathrm{MHz}$ channels. This result is due to the fact that the range in throughput is higher when using channel bonding than when not using it. In other words, STAs using channel bonding can obtain a throughput from 0 to $135 \mathrm{Mbps}$, while the upper value decreases to $65 \mathrm{Mbps}$ when channel bonding is not in use. For this reason, the disparities between STAs when using channel bonding can be higher. The fairness obtained when using two $40 \mathrm{MHz}$ channels is higher in the USA setting than in the Europe setting. Therefore, we can conclude that the advantage in throughput that can be obtained when using two $40 \mathrm{MHz}$ channels in the $2.4 \mathrm{GHz}$ Europe frequency band is at the expense of increasing the disparity between the throughputs obtained by the different STAs.

\section{Conclusions}

Channel bonding is a technique proposed in Wi-Fi networks since the standard IEEE 802.11n (Wi-Fi 4) to use wider frequency channels to be able to obtain higher throughputs.
However, its use is usually discouraged in the $2.4 \mathrm{GHz}$ frequency band, since it only allows for two $40 \mathrm{MHz}$ orthogonal (or almost orthogonal) channels. However, we found a number of limitations in previous studies on the matter, so we revised that belief for dense, uncoordinated Wi-Fi 4 environments. Our study confirms the previous consensus that it is not advisable to use channel bonding in the $2.4 \mathrm{GHz}$ frequency band with 11 channels which are $20 \mathrm{MHz}$ wide (as in North America). However, contrary to the usual assumption, we show that the use of channel bonding with $40 \mathrm{MHz}$ channels in the $2.4 \mathrm{GHz}$ frequency band with 13 or more $20 \mathrm{MHz}$ channels (the one used in many parts of the world, including Europe) results in an improvement of the average throughput achieved by STAs. Moreover, we show that, even when the number of STAs that decrease their throughput is not negligible, the improvement in throughput experienced by the "winning" STAs is much higher than the decrease in throughput experienced by the "losing" STAs. Hence, channel bonding is worth using not only from the perspective of getting a higher sum of throughputs for the network, but also from the perspective of STAs.

As the decision of using channel bonding lies in the AP but the benefits are for STAs, as a future work, we plan to shift to the STAs (therefore to the users) the decision of whether to use channel bonding or not, since these are ultimately the ones impacted by such decisions. Such a possibility will let us reach more democratic, client-centric configurations, with which we intend to address the fairness issues usually related to channel bonding. Finally, we want to explore the effects of dynamic channel bonding in the $2.4 \mathrm{GHz}$ band, since it could significantly increase the advantage of using channel bonding techniques in Wi-Fi 4.

\section{Data Availability}

The graphs used to support the findings of this study are available from the corresponding author upon request.

\section{Conflicts of Interest}

The authors declare that they have no conflicts of interest.

\section{Acknowledgments}

Jose Manuel Gimenez-Guzman, Ivan Marsa-Maestre, David Orden, and Susel Fernandez were partially funded by Project SBPLY/19/180 501/000171 of the Junta de Comunidades de Castilla-La Mancha and FEDER and by Project UCeNet (CM/JIN/2019-031) of the Comunidad de Madrid and University of Alcalá. Jose Manuel Gimenez-Guzman, Ivan Marsa-Maestre, and Susel Fernandez were also funded by Project PID2019-104855RB-I00/AEI/10.13039/ 501100011033 of the Spanish Ministry of Science and Innovation. David Orden was also partially supported by Project PID2019-104129GB-I00/AEI/10.130 39/ 501100011033 of the Spanish Ministry of Science and Innovation and by H2020-MSCA-RISE Project 734922 - CONNECT. Marino Tejedor-Romero was funded by a predoctoral contract from University of Alcalá. 


\section{References}

[1] V. Sathya, M. I. Rochman, and M. Ghosh, "Measurementbased coexistence studies of LAA \& wi-fi deployments in chicago," IEEE Wireless Communications, vol. 28, no. 1, pp. 136-143, 2021.

[2] H. A. Omar, K. Abboud, N. Cheng, K. R. Malekshan, A. T. Gamage, and W. Zhuang, "A survey on high efficiency wireless local area networks: next generation wifi," IEEE Communications Surveys \& Tutorials, vol. 18, no. 4, pp. 2315-2344, 2016.

[3] S. Chieochan, E. Hossain, and J. Diamond, "Channel assignment schemes for infrastructure-based 802.11 WLANs: a survey," IEEE Communications Surveys \& Tutorials, vol. 12, no. 1, 2010.

[4] E. de la Hoz, J. Gimenez-Guzman, I. Marsa-Maestre, and D. Orden, "Automated negotiation for resource assignment in wireless surveillance sensor networks," Sensors, vol. 15, no. 11, pp. 29547-29568, 2015.

[5] H.-J. Chen, C.-P. Chuang, Y.-S. Wang, S.-W. Ting, H.-Y. Tu, and C.-C. Teng, "Design and implementation of a clusterbased channel assignment in high density 802.11 WLANs," in Proceedings of the Network Operations and Management Symposium (APNOMS), 2016 18th Asia-Pacific, pp. 1-5, IEEE, Kanazawa, Japan, October 2016.

[6] Y. M. Kwon, K. Choi, M. Kim, and M. Y. Chung, "Distributed channel selection scheme based on the number of interfering stations in WLAN," Ad Hoc Networks, vol. 39, pp. 45-55, 2016.

[7] H. Kasasbeh, F. Wang, L. Cao, and R. Viswanathan, "Generous throughput oriented channel assignment for infrastructured WiFi networks," in Proceedings of the Wireless Communications and Networking Conference (WCNC), pp. 1-6, IEEE, San Francisco, CA, USA, March 2017.

[8] D. Orden, I. Marsa-Maestre, J. M. Gimenez-Guzman, E. de la Hoz, and A. Álvarez-Suárez, "Spectrum graph coloring to improve Wi-Fi channel assignment in a real-world scenario via edge contraction," Discrete Applied Mathematics, vol. 263, pp. 234-243, 2019.

[9] S. H. R. Bukhari, M. H. Rehmani, and S. Siraj, "A survey of channel bonding for wireless networks and guidelines of channel bonding for futuristic cognitive radio sensor networks," IEEE Communications Surveys \& Tutorials, vol. 18, no. 2, pp. 924-948, 2015.

[10] S. Barrachina-Muñoz, F. Wilhelmi, and B. Bellalta, "To overlap or not to overlap: enabling channel bonding in highdensity wlans," Computer Networks, vol. 152, pp. 40-53, 2019.

[11] L. Lanante and S. Roy, "Analysis and optimization of channel bonding in dense ieee 802.11 wlans," IEEE Transactions on Wireless Communications, vol. 20, no. 3, pp. 2150-2160, 2021.

[12] L. Deek, E. Garcia-Villegas, E. Belding, S.-J. Lee, and K. Almeroth, "The impact of channel bonding on $802.11 \mathrm{~N}$ network management," in Proceedings of the Seventh COnference on Emerging Networking EXperiments and Technologies, pp. 1-12, Tokyo, Japan, December 2011.

[13] S.-H. Lim, Y.-B. Ko, C. Kim, and N. H. Vaidya, "Design and implementation of multicasting for multi-channel multi-interface wireless mesh networks," Wireless Networks, vol. 17, no. 4, pp. 955-972, 2011.

[14] L. Deek, E. Garcia-Villegas, E. Belding, S.-J. Lee, and K. Almeroth, "Joint rate and channel width adaptation for 802.11 mimo wireless networks," in Proceedings of the 2013 IEEE International Conference on Sensing, Communications and Networking (SECON), pp. 167-175, IEEE, New Orleans, LA, USA, October 2013.
[15] J. Fang and I. T. Lu, "Efficient channel access scheme for multiuser parallel transmission under channel bonding in IEEE 802.11ac," IET Communications, vol. 9, no. 13, pp. 1591-1597, 2015.

[16] Y. Daldoul, D.-E. Meddour, and A. Ksentini, "IEEE 802.11 ac: effect of channel bonding on spectrum utilization in dense environments," in Proceedings of the 2017 IEEE International Conference on Communications (ICC), pp. 1-6, IEEE, Paris, France, May 2017.

[17] S. Barrachina-Muñoz, F. Wilhelmi, and B. Bellalta, "Dynamic channel bonding in spatially distributed high-density wlans," IEEE Transactions on Mobile Computing, vol. 19, no. 4, pp. 821-835, 2019.

[18] Z. Khan, H. Ahmadi, E. Hossain, M. Coupechoux, L. A. Dasilva, and J. J. Lehtomäki, "Carrier aggregation/ channel bonding in next generation cellular networks: methods and challenges," IEEE Network, vol. 28, no. 6, pp. 34-40, 2014.

[19] D. A. Marenda, G. M. Suranegara, S. Qamar, R. Hakimi, and E. Mulyana, "Emulating software-defined wireless network: bicasting scenario," in Proceedings of the 2017 3rd International Conference on Wireless and Telematics (ICWT), pp. 76-80, Palembang, Indonesia, July 2017.

[20] CISCO, 802.11ac: The Fifth Generation of Wi-Fi, Cisco System Technical White Paper, CISCO, San Jose, CA, USA, 2018.

[21] L. Xu, K. Yamamoto, and S. Yoshida, "Performance comparison between channel-bonding and multi-channel csma," in Proceedings of the 2007 IEEE Wireless Communications and Networking Conference, pp. 406-410, Hong Kong, China, March 2007.

[22] J. M. Gimenez-Guzman, I. Marsa-Maestre, D. Orden, E. de la Hoz, and T. Ito, "On the goodness of using orthogonal channels in WLAN IEEE 802.11 in Realistic Scenarios," Wireless Communications and Mobile Computing, vol. 2018, Article ID 5742712, 11 pages, 2018.

[23] Texas Instruments, Wlan Channel Bonding: Causing Greater Problems than it Solves, Texas Instruments, Dallas, Texas, USA, 2003.

[24] R. Chandra, R. Mahajan, T. Moscibroda, R. Raghavendra, and P. Bahl, "A case for adapting channel width in wireless networks," ACM SIGCOMM Computer Communication Review, vol. 38, no. 4, pp. 135-146, 2008.

[25] V. Shrivastava, S. Rayanchu, J. Yoonj, and S. Banerjee, "11 n under the microscope," in Proceedings of the 8th ACM SIGCOMM Conference on Internet Measurement, pp. 105-110, Greece, 2008.

[26] J. Martinez-Bauset, J. Gimenez-Guzman, and V. Pla, “Optimal admission control in multimedia mobile networks with handover prediction," IEEE Wireless Communications, vol. 15, no. 5, pp. 38-44, 2008.

[27] A. M. Voicu, L. Lava, L. Simić, and M. Petrova, "The importance of adjacent channel interference: experimental validation of ns-3 for dense wi-fi networks," in Proceedings of the 20th ACM International Conference on Modelling, Analysis and Simulation of Wireless and Mobile Systems, pp. 43-52, Miami, FL, USA, November 2017.

[28] Rec ITU-R P 1238-8, Propagation Data and Prediction Methods for the Planning of Indoor Radiocommunication Systems and Radio Local Area Networks in the Frequency Range $300 \mathrm{MHz}$ to $100 \mathrm{GHz}$, International Telecommunication Union, Geneva, Switzerland, 2015.

[29] T. Chrysikos, G. Georgopoulos, and S. Kotsopoulos, "Sitespecific validation of itu indoor path loss model at $2.4 \mathrm{GHz}$," in Proceedings of the 2009 IEEE International Symposium on a 
World of Wireless, Mobile and Multimedia Networks \& Workshops, pp. 1-6, IEEE, Kos, Greece, 2009.

[30] Mukta and N. Gupta, "Analytical approach towards available bandwidth estimation in wireless Ad Hoc networks," Wireless Networks, vol. 26, no. 10, pp. 1-26, 2020.

[31] IEEE Computer Society, "IEEE standard for information technology- local and metropolitan area networks- Specific requirements- Part 11: Wireless LAN Medium Access Control (MAC) and Physical Layer (PHY) specifications amendment 5: enhancements for higher throughput," in Proceedings of the IEEE Std 802.11n-2009 (Amendment To IEEE Std 802.11-2007 as Amended by IEEE Std 802.11k-2008, IEEE Std 802.11r-2008, IEEE Std 802.11y-2008, and IEEE Std 802.11w-2009), pp. 1-565, 2009.

[32] M. Kim and C.-H. Choi, "Hidden-node detection in ieee 802.11n wireless LANs," IEEE Transactions on Vehicular Technology, vol. 62, no. 6, pp. 2724-2734, 2013.

[33] E. De La Hoz, I. Marsa-Maestre, J. M. Gimenez-Guzman, D. Orden, and M. Klein, "Multi-agent nonlinear negotiation for Wi-Fi channel assignment," in Proceedings of the 16th Conference on Autonomous Agents and MultiAgent Systems, pp. 1035-1043, Sao Paulo, Brazil, 2017.

[34] C. Camacho-Gómez, I. Marsa-Maestre, J. M. GimenezGuzman, and S. Salcedo-Sanz, "A coral reefs optimization algorithm with substrate layer for robust wi-fi channel assignment," Soft Computing, vol. 23, no. 23, pp. 12621-12640, 2019.

[35] I. Marsa-Maestre, E. de la Hoz, J. M. Gimenez-Guzman, D. Orden, and M. Klein, "Nonlinear negotiation approaches for complex-network optimization: a study inspired by wi-fi channel assignment," Group Decision and Negotiation, vol. 28, no. 1, pp. 175-196, 2019.

[36] M. Achanta, "Method and apparatus for least congested channel scan for wireless access points," US Patent App, 2006.

[37] G. F. Riley and T. R. Henderson, "The Ns-3 Network Simulator," in Modeling and Tools for Network Simulation, pp. 15-34, Springer, Berlin, Germany, 2010.

[38] R. K. Jain, D.-M. W. Chiu, and W. R. Hawe, "A quantitative measure of fairness and discrimination," Eastern Research Laboratory, Digital Equipment Corporation, Hudson, MA, USA, 1984. 